\title{
A new method to determine air shower propagation direction based on radio signal patterns
}

\author{
Mohammad Sabouhi ${ }^{1}$ \\ Department of Physics, Semnan University \\ Semnan, Iran \\ E-mail:m.sabouhi@semnan.ac.ir

\section{Gohar Rastegarzadeh} \\ Department of Physics, Semnan University \\ Semnan, Iran \\ E-mail: grastegaresemnan.ac.ir
}

\begin{abstract}
The propagation direction is one of the key parameters of an air shower. In this study we introduce a new and unique approach to determine propagation direction of an air shower based on filtered peak radio amplitudes which can shape radio signal patterns. For this purpose, a series of simulations were performed with CORSIKA and CoREAS software. Simulated air showers initiate from Proton and Iron primary particles with $10^{17} \mathrm{eV}$ energy and have different zenith angles from vertical to $60^{\circ}$. Time based results were converted and analyzed using a specially designed computer code to calculate the peak radio amplitudes of different electric field components $\left(E_{x}, E_{y}, E_{z}\right)$ in the 32-64 MHz frequency band. We investigate four different shower propagation directions including North-South and East-West orientations in this paper. It is found that the radio signal patterns of different electric fields components especially the North and Vertical Components can be used to determine an air shower propagation direction accurately. This new technique is especially useful for inclined air showers where the impact of an air shower propagation direction is very prominent on its radio signal patterns.
\end{abstract}

The 34th International Cosmic Ray Conference

30 July- 6 August, 2015

The Hague, The Netherlands

\section{${ }^{1}$ Speaker}




\section{Introduction}

One of the key parameter of an air shower which is always a subject of study is the direction which the shower enters the earth atmosphere. For ultra-high energy cosmic rays this direction can be used to determine the possible source candidates of a cosmic ray since higher energy particles deflect less by galactic and extragalactic magnetic fields [1],[2]. At this moment there are some available approaches to determine an air shower propagation direction [3],[4]. These methods are based on current techniques to investigate an air shower characteristics. Although these approaches are in used for many years, there has been always an explore for a new and better detection technique which could complement the existing particle detectors and air fluorescence telescope. This new approach should have been less affected by environmental changes besides having a high duty cycle. Radio detection of cosmic ray air showers can provide such properties. It is for this reason that we see a revive in radio detection technique in recent years [5],[6],[7],[8]. This new approach has already been used to determine some of the key characteristics of an air shower including the arrival direction based on time measurements [9]. Simulation of radio emission from cosmic ray sir showers plays a key role in making radio detection a reliable approach [10],[11]. In this paper we introduce a new method to determine an air shower propagation direction based on radio signal patterns by using radio detection technique and Monte Carlo simulations of geosynchrotron radio emission [12]. This new method shows just one of the advantages of radio detection of cosmic ray air showers. Analyzing radio signals not only allow us to estimate the propagation direction but also give us many other possible information about the most important air shower characteristics like the initial energy and type of primary particle[13]. It should be noted that some parameters do have huge impact on radio signals such as the zenith angle with which the shower enters the earth atmosphere and the earth magnetic field values. We'll discuss the importance of the later one in an upcoming study.

\section{Methodology}

The results presented in this article are based on CORSIKA 7.4 [14] and CoREAS 1.0 [15] simulations. We have done a series of simulations with proton as a primary particle with $10^{17} \mathrm{eV}$ initial energy. We use QGSJETII-03 [16],[17] as the hadronic interaction model with thinning set to $10^{-6}$ and the earth magnetic field correspond with the Pierre Auger observatory located in Argentina [18]. A radio array consist of 73 radio antennas measure radio signals from shower core to 100 meters. Our focus in this study is on radio signals around shower core since they become so weak in long distances that may not have useful values especially for low energy air showers [19]. The raw time-dependent values of electric field components converted into frequency- dependent values and the peak radio amplitudes in the 32-64 MHz frequency band is obtained for North, West and Vertical directions using a specially designed computer code. These peak radio amplitude values which has been obtained from all 73 virtual radio antennas for all components can shape radio signal patterns in contour format. These shapes and their orientations form the basis of our discussion. First we discuss the radio signal patterns for vertical air showers followed by inclined air showers. Although we have obtained radio signal patterns for all four components including North, West, Vertical and Total parts, two of them are more important in the following discussions. 


\section{Vertical Air showers}

We start our study by analyzing the radio signal patterns for a vertical shower. The West component under the effect of the earth magnetic field and East-West effect [20] always have an orientation to the East for a shower with this specific property. In other hand the North and Vertical components have valuable information about the arrival direction of a vertical air shower.

Fig. 1 shows the North component shapes for a vertical air shower. These patterns are almost symmetric around the $\mathrm{Y}$ axis and the radio antennas have measured almost the same values on the both side of the $\mathrm{Y}$ axis. The $E_{z}$ component figure, is completely different. Fig. 2 shows how the highest values have been measured by the radio antennas located at the center of the air shower. The values decreases rapidly as we move from shower core. These two type of figures are almost identical for vertical showers regardless of their initial energy and type of primary particle and only depends on some shower property like the earth magnetic field parameter as we'll discuss in an upcoming study. So it is possible to determine the propagation direction of a vertical air shower just by analyzing the $E_{x}$ and $E_{z}$ patterns.
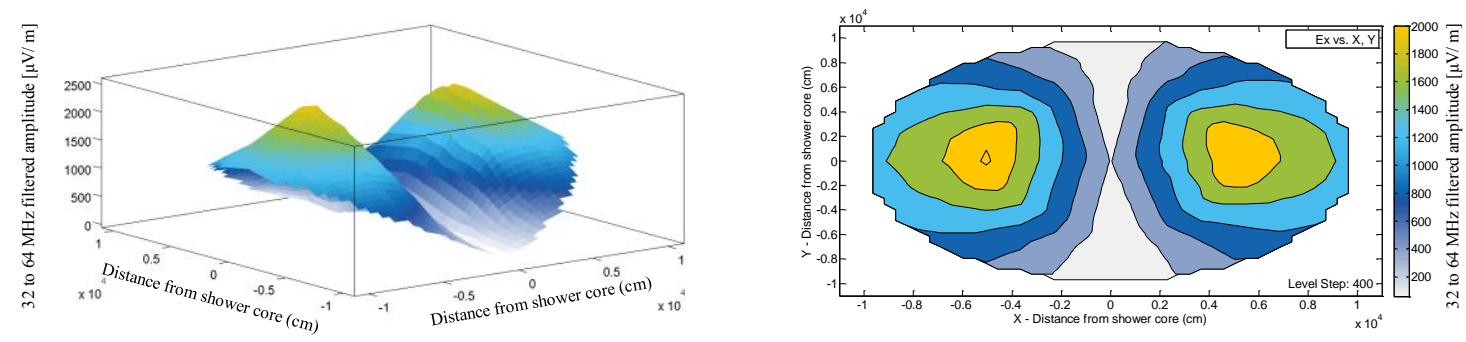

Figure 1: From left to right: 3D plot of the peak radio amplitude values in the North direction for a vertical proton air shower with $10^{17} \mathrm{eV}$ initial energy and the same figure in contour format.
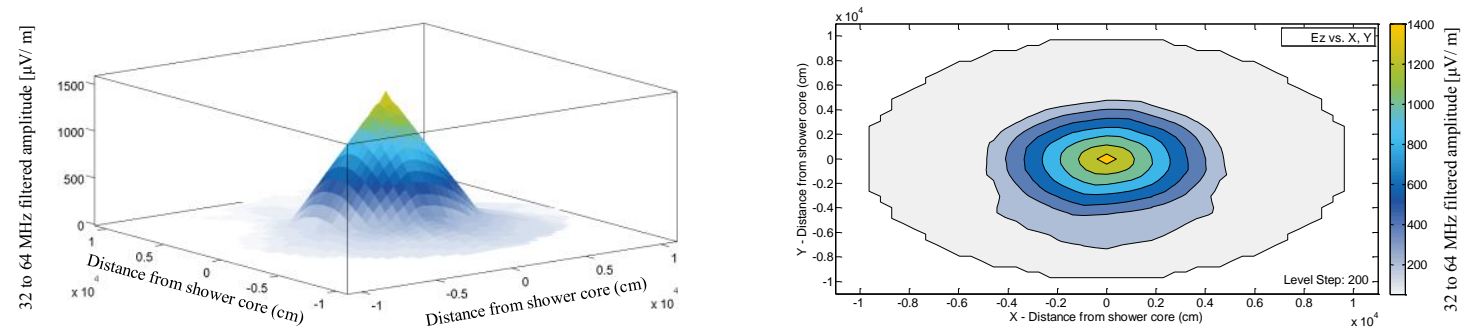

Figure2: From left to right : 3D plot of the peak radio amplitude values in the Vertical direction for a vertical proton air shower with $10^{17} \mathrm{eV}$ initial energy and the same figure in contour format.

As we mentioned, the overall shape of these patterns are identical for all vertical showers regardless of the type of primary particle or initial energy. Fig. 3 shows the radio signal patterns in North and Vertical directions for a vertical Iron shower with $10^{17} \mathrm{eV}$ energy. It is clear that these shapes are almost equal to the ones showcased in Fig. $1 \& 2$. 

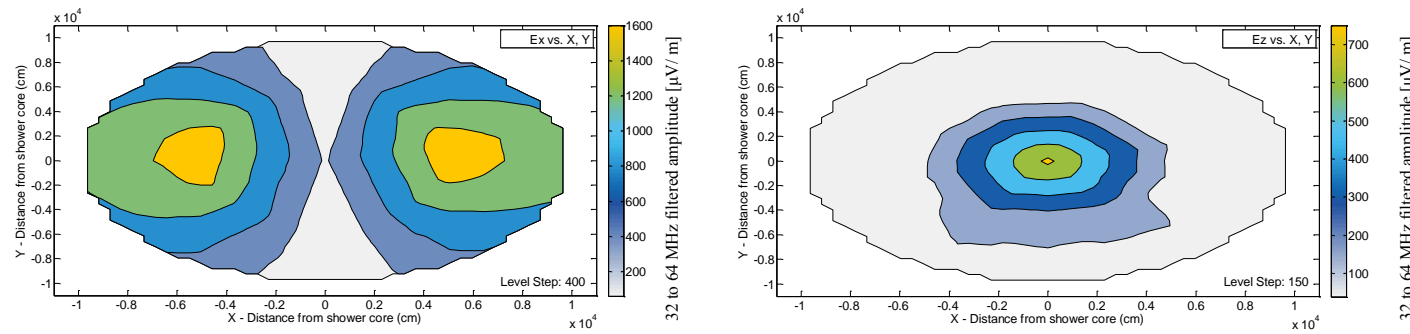

Figure3: From left to right : Peak radio amplitude values in the North and Vertical directions for a vertical Iron air shower with $10^{17} \mathrm{eV}$ initial energy and the same figure in contour format.

\section{Inclined Air Showers}

As a shower becomes inclined the zenith angle of the shower shows its impact on all radio signal patterns especially in the North and Vertical directions. In this section we investigate inclined air showers which are propagating into different orientations.

\subsection{North-South Air Showers}

For the North-South air showers we start with an inclined air shower which is propagation to the South with $\theta=30^{\circ}$. For North-South air showers both $E_{x}$ and $E_{z}$ radio signal patterns forms similarly so we can determine an air shower propagation direction only by considering one of these two patterns.

For inclined air showers and as the zenith angle increases, the radio signal patterns in the North and Vertical directions form differently compared to vertical air shower patterns. In this case the highest values were measured by the radio antennas located in the South where the shower is actually propagating.

Fig.4 shows the impact of an air shower zenith angle on radio signal patterns. The $E_{z}$ pattern is also identical to the $E_{x}$ and it is heading towards the South. Both figures have lost their symmetric property as the shower has become inclined.
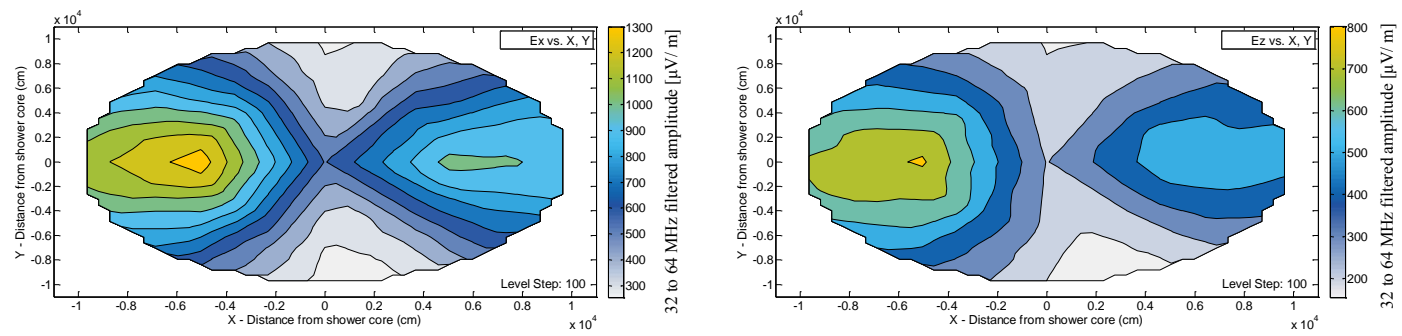

Figure4: From left to right: Contour plot of radio signal patterns in the North and Vertical directions for an air shower with $\theta=30^{\circ}, \varphi=180^{\circ}$. For inclined air showers propagation to the North or South, both patterns share same orientations generally. 
As the zenith angle increases to $45^{\circ}$ the $E_{x}$ and $E_{z}$ patterns form more apparently in the direction where an air shower is propagating. Fig. 5 shows how these patterns actually behave in a contour format. Both $E_{x}$ and $E_{z}$ figures are also similar in this situation.
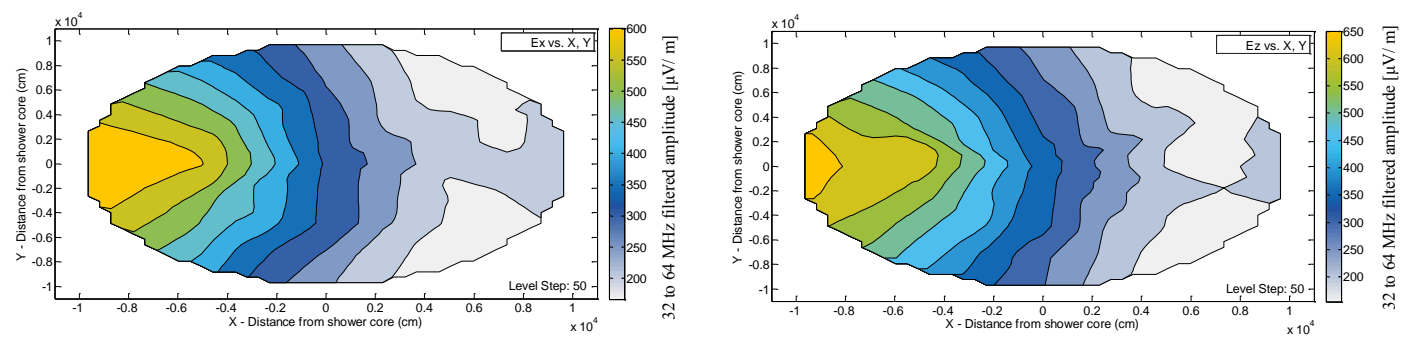

Figure5: From left to right: $E_{x}$ and $E_{z}$ patterns for an inclined air shower with $\theta=45^{\circ}, \varphi=180^{\circ}$

In the final step of analyzing North-South showers, we investigate peak radio amplitude patterns for two fully inclined air showers with $\theta=60^{\circ}$ which are propagating to the South and North. The $E_{x}$ pattern tends more than ever to the propagation direction of the air showers. As we expect, the $E_{z}$ pattern also tends towards the direction where the shower is propagating. Fig. 6 shows the high impact of a shower zenith angle on the radio emission patterns. By looking to these patterns, their directions and the peak radio amplitude values, we can actually determine the propagation direction of an air shower.
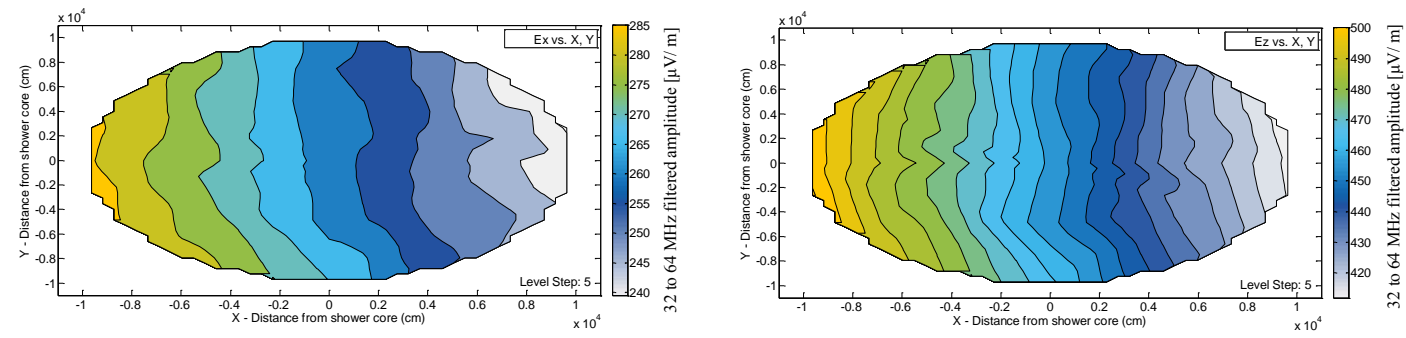

Figure6: From left to right: $E_{x}$ and $E_{z}$ patterns for an inclined air shower with $\theta=60^{\circ}, \varphi=180^{\circ}$

This type of tendency toward the shower propagation direction is also present for showers which are propagating to the North. Fig. 7 shows the exact same behavior but in the opposite direction for an inclined air shower propagating in the North direction.
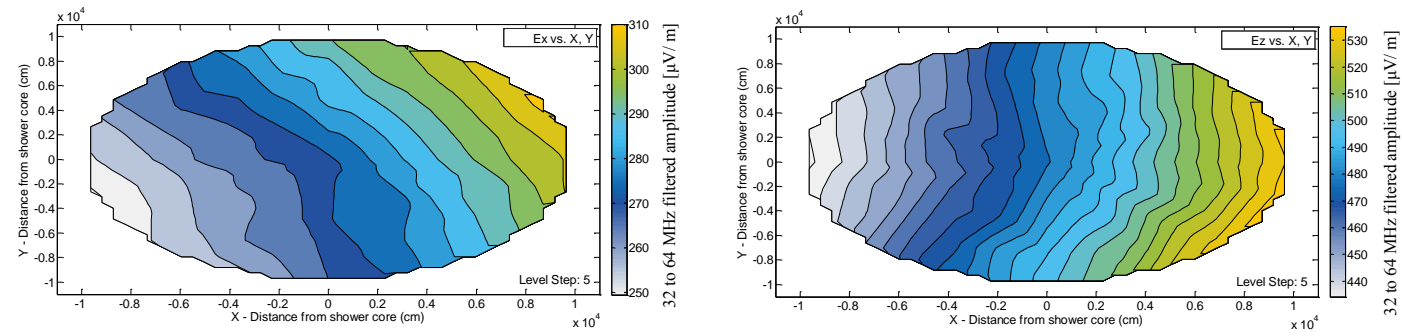

Figure7: From left to right: $E_{x}$ and $E_{z}$ patterns for an inclined air shower with $\theta=60^{\circ}, \varphi=0^{\circ}$ 
Figures 2 to 6 clearly shows the impact of the zenith angle on the radio signal patterns. As the shower becomes more inclined the emerging patterns tend more and more in the propagation direction of an air shower in the North-South orientations.

\subsection{East-West Air Showers}

As we discussed in the previous section, for a North-South shower only one of the peak radio amplitude patterns from $E_{x}$ or $E_{z}$ components was enough to determine an air shower propagation direction because both shared same orientations towards the shower propagating direction. However for the East-West case we need to pay attention to both $E_{x}$ and $E_{z}$ peak radio amplitude patterns separately. The combination of these two patterns can help us to determine the propagating direction of an air shower.

We start our investigation by analyzing a very inclined shower which is propagating to the West. In this case and as the shower is propagating to the West, the $E_{x}$ pattern tends toward North. The $E_{z}$ pattern shapes towards East, which is the opposite orientation of the shower propagation direction as it can be seen in Fig8. The combination of these two type of pattern orientations are unique for an air shower which is propagating to the West and as a result it can be used to determine an air shower propagation direction.
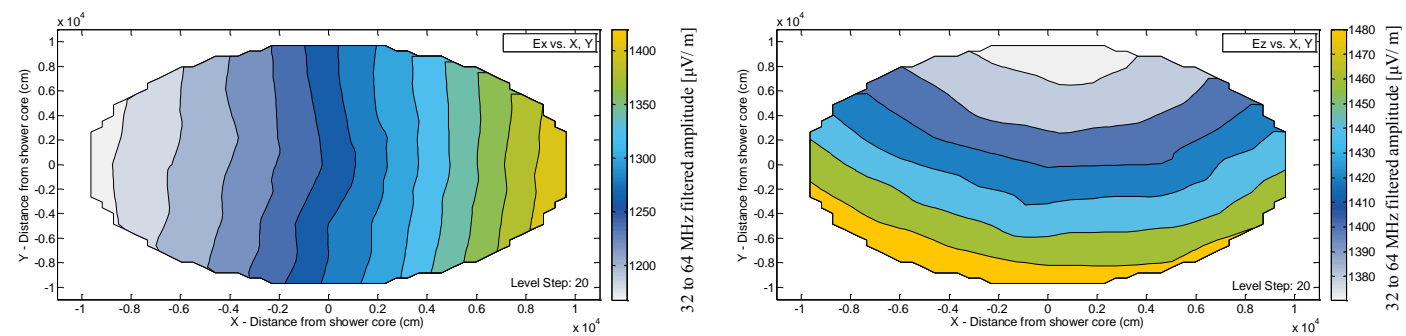

Figure8: From left to right: $E_{x}$ and $E_{z}$ patterns for an inclined air shower with $\theta=60^{\circ}, \varphi=90^{\circ}$

In the next step, we investigate an air shower which is propagating to the East. Fig.9 shows the combination of the two peak radio amplitude patterns for that air shower. In this case the $E_{x}$ pattern heads to the South where the $E_{z}$ figure remains unchanged in comparison to a shower which is propagating to the West. So we are looking at a very unique combination orientations in each case.
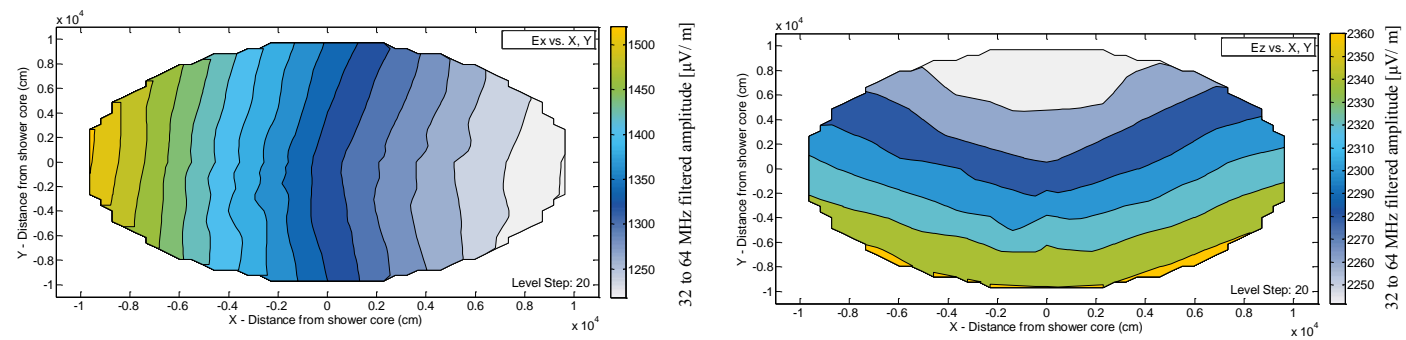

Figure9: From left to right: $E_{x}$ and $E_{z}$ patterns for an inclined air shower with $\theta=60^{\circ}, \varphi=270^{\circ} 0$ 


\section{Conclusions}

Based on CORSIKA and CoREAS code, we introduced a new method to determine one of the key parameter of an air shower which is the propagation direction. We showed that for a vertical air shower the $E_{x}$ and $E_{z}$ components form a very unique patterns regardless of the $\varphi$ parameter value. The $E_{x}$ pattern is almost symmetric to the $\mathrm{Y}$ axis while the $E_{z}$ pattern is isotropic in all directions for a vertical shower. These type of radio patterns from radio signals are universal for all type of vertical showers regardless of their initial energy and type of primary particles and only depends on some parameters like the earth magnetic field values.

This situation changes as the shower becomes inclined. For inclined air showers which are propagating in the North-South Directions, $E_{x}$ and $E_{z}$ patterns tends towards the propagation direction of the air showers similarly. This tendency increases as the zenith angle increases. For the East-West showers the two patterns act differently. When a shower is propagating to the west direction, the $E_{x}$ patterns shifts towards North while the highest values of the $E_{z}$ patterns measures in the East direction. For a shower which is propagating to the East direction, the $E_{z}$ pattern remains unchanged and tends towards East but the $E_{x}$ pattern heads towards South. Despite of differences mentioned above there is always a unique combination of $E_{x}$ and $E_{z}$ pattern orientations for an air shower propagation in a specific direction. The combination of these two pattern directions can be used to determine an air shower propagation.

\section{References}

[1] Pierre Sokolsky., Introduction to Ultra High Energy Cosmic Ray Physics, Addison-Wesley, 1989.

[2] Chad Barrett Finley., Anisotropy of Arrival Directions of Ultrahigh Energy Cosmic Rays, Master Thesis, Columbia University, 2006.

[3] R. Abbasi et al., Measurment of the Anisotropy of Cosmic-Ray Arrivial Directions with ICECUBE, 2010 ApJ 718 L194.

[4] A. Aab et al., Searches for Large-Scale Anisotropy in the Arrival Directions of Cosmic Rays Detected above Energy of $1019 \mathrm{eV}$ at the Pierre Auger Observatory and the Telescope Array, ApJ, 794, 172 (2014).

[5] H. Falcke, W. D. Apel, A. F. Badea et al., Detection and imaging of atmospheric radio flashes from cosmic ray air showers, Nature 435 (2005) 313-316.

[6] T. Huege, W. D. Apel, T. Asch et al., Radio detection of cosmic ray air showers with LOPES, Nuclear Physics B (Proc. Suppl.) 165 (2007) 341-348.

[7] D. Ardouin, A. Bell'etoile, D. Charrier et al., Radio-detection signature of high-energy cosmic rays by the CODALEMA experiment, Nucl. Instr. Meth. A 555 (2005) 148-163.

[8] A. van den Berg et al., Radio detection of high-energy cosmic rays at the Pierre Auger Observatory, in: Proceedings of the 30th ICRC, Merida, Mexico, 2007, astro-ph/0708.1709. 
[9] Karlsruhe (1998).T. Huege, M. Ludwig, C.W. James, Simulating radio emission from air showers with CoREAS, AIP Conf. Proc. 1535, 128-132 (2013), doi:10.1063/1.4807534.

[10] Huege, T., Falcke, H., Radio emission from cosmic ray air showers. Monte Carlo simulations, Astronomy and Astrophysics, v.430, p.779-798 (2005).

[11] Huege, T., Falcke, H., Radio emission from cosmic ray air showers: Simulation results and parametrization, 2005, Astropart. Physics 24, 116-136.

[12] Huege, T., Ulrich, R., Engel, R., Monte Carlo simulations of geosynchrotron radio emission from CORSIKA-simulated air showers, 2007, Astropart. Physics 27, 392-405.

[13] Huege, T., Ulrich, R., Engel, R., Dependence of geosynchrotron radio emission on the energy and depth of maximum of cosmic ray showers, 2008, Astropart. Physics 30, 96-104.

[14] D. Heck, J. Knapp, J.N. Capdevielle, G. Schatz, T. Thouw, CORSIKA: A Monte Carlo Code to Simulate Extensive Air Showers, FZKA Report 6019 (1998), Forschungszentrum.

[15] Karlsruhe (1998).T. Huege, M. Ludwig, C.W. James, Simulating radio emission from air showers with CoREAS, AIP Conf. Proc. 1535, 128-132 (2013), doi:10.1063/1.4807534.

[16] S. Ostapchenko., Non-linear screening effects in high energy hadronic interactions, Phys. Rev. D74 (2006) 014026.

[17] S. Ostapchenko., On the re-summation of enhanced Pomeron diagrams, Phys. Lett. B636 (2006) $40-45$.

[18] J. Abraham, M. Aglietta, I.C. Aguirre, et al., Properties and performance of the prototype instrument for the Pierre Auger Observatory, Nucl. Instrum. Meth. A523 (2004) 50-95.

[19] F. G. Schröder, Instruments and Methods for the Radio Detection of High Energy Cosmic Rays, Springer Theses, DOI: 10.1007/978-3-642-33660-7_2, (C) Springer-Verlag Berlin Heidelberg 2012.

[20] B. Bartoli et al. (ARGO-YBJ Collaboration), Evidence of a geomagnetic effect on extensive air showers detected with the ARGO-YBJ experiment, Phys. Rev. D 89, 052005 - Published 28 March 2014. 